\title{
Severidad en las dificultades de aprendizaje de la lectura: diferencias en la percepción del habla y la conciencia fonológica
}

\section{Severity of Reading Difficulties: Individual Differences in Speech Perception and Phoological Awareness}

\author{
Juan L. Luque, Soraya Bordoy, Almudena Giménez, Miguel López-Zamora, Victoria Rosales \\ Departamento de Psicología Evolutiva y de la Educación. Facultad de Psicología. Universidad de Málaga, España.
}

Disponible online 31 de agosto de 2011

\begin{abstract}
Un conjunto de tareas de conciencia fonológica, identificación y discriminación de fonemas y juicios de orden temporal fue administrada a una muestra de niños disléxicos y sus respectivos grupos de control edad (CE) y control nivel lector (CNL). Los estímulos de las tareas de identificación y discriminación fueron nueve sílabas de un contraste de lugar de articulación en el continuo /ba-da/, construidas mediante la manipulación de los formantes 2 y 3 . Los datos fueron analizados siguiendo dos métodos. Las comparaciones ANOVA mostraron diferencias significativas en las tareas de discriminación y juicio de orden temporal entre los disléxicos y los grupos controles, mientras que los parámetros de la tarea de identificación (pendiente y límite categorial) no alcanzaron la significación. Se desarrolló también un procedimiento para evaluar la desviación. El 44\% de los niños disléxicos mostraron al menos dos medidas con una desviación estándar de 1.65. Además, hubo una relación entre las ejecuciones en conciencia fonológica y percepción del habla. En general los resultados fueron muy similares a los principales hallazgos descritos en la bibliografía. Por tanto, defendemos que las medidas procedentes de las tareas de percepción del habla podrían ser incorporadas como un elemento complementario en el procedimiento de diagnóstico de la dislexia evolutiva.
\end{abstract}

Palabras Clave: Dislexia; Percepción del Discurso; Consciencia Fonológica; Phonological Awareness, Diagnóstico.

\begin{abstract}
A set of phonological awareness, phoneme identification, discrimination and temporal order tasks were administered to dyslexic children and chronological age (CA) and reading-level (RL) comparison groups. The identification and discrimination task stimuli consisted of nine/ba-da/ syllables over an articulation continuum. Syllables were obtained by manipulating formants 2 and 3. Data were analyzed using two methods. ANOVA comparisons showed significant differences between the dyslexic and control groups in the discrimination and temporal order tasks, whereas parameters from the identification task (slope and boundary) did not reach significance. A procedure to assess deviance was also developed. $44 \%$ of dyslexic children showed at least two 1.65 standard deviation measures. Moreover, there was an association between phonological awareness and speech perception performance. Generally, the results were similar to the main findings described in the literature. Thus, we suggest that measures from speech perception tasks could be incorporated as complementary elements in developmental dyslexia diagnosis procedures.
\end{abstract}

Keywords: Dyslexia; Speech Perception; Phonological Awareness; Diagnosis.

Debemos agradecer a los centros escolares: San Roque, de Tolox; Huertas Viejas, de Coín; Arquitecto Sánchez Sepúlveda, de Alozaina y el Lex Flavia de Málaga por su estimable colaboración. Del mismo modo, agradecemos al profesor Pedro Cobos por el desarrollo del programa en Visual Basic que permite calcular los parámetros asociados a la prueba de identificación.

Correspondencia: Juan Luis Luque Villaseca. Departamento de Psicología Evolutiva y de la Educación. Facultad de Psicología. Campus de Teatinos, s/n. Edificio de Psicología y Ciencias de la Educación. 29071-Málaga. E-mail: juan.luque@uma.es. E-Mails de los otros autores: Soraya Bordoy: soribordoy@gmail.com; Almudena Giménez: Almudena@uma.es; Miguel López-Zamora: migueljar@gmail.com; Victoria Rosales: victoria.rosa1es773@gmail.com 


\section{Introducción}

Se están produciendo avances significativos en la identificación temprana y el diagnóstico de la dislexia evolutiva. Respecto a la identificación temprana puede destacarse el desarrollo de metodologías de respuesta a la intervención (RtI: Response to Intervention; véase Jiménez, Baker, Rodríguez, Crespo, Artiles y Alfonso, 2011; Jiménez, 2010) y metodologías de evaluación basadas en el curriculum (CBM o CBA: curriculumbased measurement or assessment; propuestas originalmente por Deno, 1987). La combinación de estas metodologías está teniendo consecuencias muy positivas (Fuchs y Fuchs, 2006). Aunque no con tanta intensidad, también se han dado pasos importantes para mejorar el diagnóstico de la dislexia. Tal y como afirman Shaywitz, Morris y Shaywitz (2008), "la dislexia es algo más que una simple puntuación en un test de lectura" y su diagnóstico debe ser realizado por un clínico con experiencia, capaz de valorar el desequilibrio entre las tareas que implican habilidades fonológicas y las que implican otras capacidades cognitivas y lingüísticas. La estrategia fundamental en este sentido ha sido incorporar pruebas de carácter etiológico, es decir, tareas procedentes de la investigación sobre las causas de la dislexia, al proceso de diagnóstico clínico (Marzola y Shepherd, 2005; Shaywitz, 2003; Snowling, 2000).

Estas tareas responden principalmente a la hipótesis fonológica básica (Stanovich, 1988; "the phonological-core variable differences model"). Se ha venido considerando que el déficit fonológico básico está asociado a tres grandes áreas (Wagner y Torgesen, 1987): la conciencia fonológica (Bradley y Bryant, 1978; Bruck, 1992; Manis, Custodio y Szeszulski, 1993), la recodificación fonológica durante el acceso léxico (Bowers y Swanson, 1991; Denckla y Rudel, 1976; Wolf, 1986), y la memoria fonológica, operativa y/o a corto plazo (Byrne y Shea, 1979; Shankweiler, Liberman, Mark, Fowler, y Fischer, 1979). Estas áreas han sido incorporadas con éxito en baterías especialmente diseñadas para identificar y diagnosticar la dislexia evolutiva (véase la Batería francesa EVALEC de SprengerCharolles Colé, Béchennec, y Kipffer-Piquard, 2005; la Batería española DIS-ESP, Carrillo y Alegría, 2009; Carrillo, Alegría, Miranda y Sánchez, 2011).

Sin embargo, estas tareas no cubren el amplio rango de hipótesis etiológicas que podrían contribuir al diagnóstico de la dislexia. Ramus, Rosen, Dakin, Day, Castellote, White y Frith (2003) utilizaron probablemente la mayor batería de pruebas fonológicas, auditivas, visuales, cerebelares y motoras que se haya administrado a una muestra de disléxicos. A pesar del ciclópeo esfuerzo de estos autores tampoco consiguieron completar la gama de hipótesis, tareas y medidas descritas en la bibliografía especializada. El asunto no es sólo una cuestión cuantitativa. Tal y como se ha puesto de manifiesto en varios de los artículos de este monográfico, la dislexia podría tener rasgos específicos que la distingan de otros trastornos (Serniclaes, 2011), sin embargo, la variabilidad de perfiles y síntomas asociados son también una constante. Los disléxicos presentan perfiles diferenciados según la ortografía y la experiencia lectora (Sprenger-Charolles, 2011). Los perfiles individuales son variados, estando su severidad y la comorbilidad con otros trastornos probablemente ligados a causas genéticas (Pernet, Dufor y Démonet, 2011). Finalmente, la mayoría de los niños disléxicos, junto con el déficit fonológico básico, presentan déficit en otros dominios, lo que ha llevado a Pennington (2006) a defender un modelo de déficit múltiple. Parece por tanto razonable y necesario continuar la transferencia de las tareas utilizadas en la investigación a la práctica clínica, más allá de las pruebas derivadas de la hipótesis fonológica básica. De esta forma, ampliaríamos el arsenal de herramientas para afinar diagnósticos individualizados.

El objetivo fundamental de este estudio piloto es desarrollar y testar un conjunto de estas tareas originalmente asociadas a la hipótesis de la percepción auditiva rápida (Tallal, 1980). En concreto, nos proponemos replicar algunos de los resultados más representativos de la bibliografía especializada respecto a tres tareas, identificación, discriminación y juicio de orden temporal de sonidos tipo habla, siguiendo un estudio ya clásico (Reed, 1989). El estudio posee además el valor de presentar resultados en español, ya que hasta donde sabemos, sólo muy recientemente se han aplicado a muestras de disléxicos españoles pruebas similares (Luque, Serniclaes, López-Zamora, Bordoy, Giménez, Rosales y Varona, 2011).

Las razones que nos han llevado a seleccionar estas tres pruebas del amplio abanico de pruebas experimentales existentes son de son de dos tipos: plausibilidad teórica y alcance práctico. Según la International Dyslexia Association, "la dislexia es una dificultad específica del aprendizaje que es neurobiológica en su origen. Está caracterizada por dificultades de exactitud y fluidez en el reconocimiento de palabras, dificultades en el deletreo y en las habilidades de decodificación. Estas dificultades provienen típicamente de un déficit en el componente fonológico del lenguaje que es a menudo inesperado en relación a otras habilidades cognitivas y a la provisión de una instrucción escolar efectiva" (Lyon, Shaywitz y Shaywitz, 2003). Existe un amplio consenso sobre el origen fonológico de la dislexia (Snowling, 2000), respaldado en una enorme cantidad de evidencia empírica que señala a las dificultades fonológicas como la causa más robusta y específica de la dislexia, tanto en niños como en adolescentes disléxicos (Fletcher et al. 1994; Morris et al., 1998; Shaywitz et al. 1999; Stanovich y Siegel 1994). El impacto del déficit fonológico resulta crítico para el aprendizaje de las correspondencias entre letras y sonidos, sin embargo, se extiende más allá de un déficit estrictamente de decodificación (Luque, López-Zamora y Álvarez, 2011).

Ahora bien, si el origen de la dislexia es un trastorno neurobiológico de origen genético que está presente desde el nacimiento, entonces es razonable preguntarse ¿cuáles son las causas primeras de este déficit fonológico básico? Diversos autores han defendido que algún tipo de déficit relativo al procesamiento y/o percepción auditiva podría ser la causa más 
lógica y parsimoniosa para explicar el desarrollo alterado de las representaciones fonológicas (Boets, Wouters, Wieringen y Ghesquière, 2007; Goswami, 2003). Numerosos estudios han confirmado la presencia de déficit auditivos en los disléxicos, además, a través de una amplia variedad de tareas (Boets et al., 2007; Manis et al. 1997; Ramus, 2003).

Siguiendo a McBride-Chang (1995), el modelo teórico que implícitamente manejan muchos autores podría ser el siguiente. Primero, las habilidades perceptivas del habla de carácter innato emergen y son refinadas durante el desarrollo temprano (Khul, 2004; Serniclaes, 2011). Existen diferencias significativas en el desarrollo fonológico durante el primer año de vida entre bebés en riesgo de padecer dislexia y muestras controles (Molfese, 2000; Richardson, Leppänen, Leiwo y Lyytinen, 2003; van Leeuwen, Been, van Herten, Zwarts, Maassen, y van der Leij, 2007). Segundo, este desarrollo temprano es la base del posterior desarrollo fonológico y podría explicar las diferencias individuales que se detectan durante el segundo y tercer año de vida (Lytinen et al., 2004; Scarborough, 1990). Finalmente, como Werker y Tees (1987) defendieron, pequeñas diferencias en la percepción del habla podrían tener un impacto considerable durante la fase de aprendizaje de la lectura. Si las categorías fonológicas son poco robustas, éstas podrían sufrir bajo situaciones exigentes, como serían los inicios del aprendizaje de la lectura, donde la segmentación de las palabras en fonemas y el aprendizaje de las correspondencias de estos con las letras se constituyen en un desafío.

El esfuerzo por concretar cuáles son estas diferencias perceptivas y qué efectos producen sobre el desarrollo fonológico no ha cesado hasta la actualidad. Según una reciente propuesta, el stress o dificultad que experimentan los niños disléxicos estaría provocado por el tamaño de las unidades fonológicas que conforman su repertorio (Grain Size Theory: Ziegler y Goswami, 2005). Si estas unidades no han alcanzado con nitidez el "calibre" de fonema, entonces, esto condicionaría la ejecución de las tareas de conciencia fonémica y, consecuentemente, el aprendizaje de las reglas de conversión grafemafonema. Por otra parte, se pretende especificar en qué consiste el principal déficit perceptivo de los disléxicos. En este Monográfico se presenta la teoría de la percepción del modo alofónico (Serniclaes, 2011, Serniclaes et al. 2004) según la cual el problema principal estaría relacionado con el desarrollo del sistema fonológico, en concreto con la transición entre un sistema de percepción universal de los sonidos tipo habla al sistema específico de cada lengua. La alteración de este proceso haría que los sujetos disléxicos conservaran las categorías fonéticas universales. Esta superabundancia de categorías dificultaría directamente el aprendizaje de las reglas de conversión, dificultando la proyección de grafemas a fonemas.

Por tanto, la primera razón para seleccionar estas tareas es porque representan un marco teórico desde el que tenazmente se ha investigado la naturaleza del déficit fonológico temprano y sus consecuencias para el desarrollo fonológico posterior y el aprendizaje de la lectura. Pero además, a partir de estas tres tareas se puede calcular un conjunto de medidas que abarcan un rango amplio de posibilidades relativas a las propiedades perceptivas de la fonología de nuestras lenguas.

En concreto, cuatro son las hipótesis relativas a la percepción auditiva que se han propuesto. La hipótesis del procesamiento auditivo rápido (Tallal, 1980), en la que fundamentalmente se centra este estudio piloto, intentando replicar los resultados de Reed (1989). Vamos a prestar especial atención a las puntuaciones a partir de un criterio de desviación, dado su potencial valor como herramienta diagnóstica en la práctica clínica. Siguiendo este procedimiento, se ha encontrado déficit de procesamiento auditivo rápido en un rango que va desde 0 al 50\% de los participantes disléxicos. Debe además señalarse, que los altos porcentajes se producen en estudios que utilizan un amplio rango de tareas y medidas (véase Ramus, 2003, para un revisión crítica), superior al que se utilizará en este piloto. En segundo lugar, la hipótesis del retraso o debilidad en la percepción categorial (Brandt y Rosen, 1980). Un conjunto de medidas relativas a la percepción categorial de los sonidos tipo habla han mostrado, en general, que los disléxicos presentan menor agudeza para diferenciar entre categorías, así como categorías más débilmente separadas. Estos resultados han aparecido hasta en un $75 \%$ de estudios, según Serniclaes et al. (2004; Serniclaes, 2006). En este estudio piloto tomaremos las medidas de pendiente y límite categorial procedentes de la prueba de identificación y estudiaremos las diferencias de la curva de discriminación. Las dos anteriores hipótesis han sido además criticadas por no representar déficit específicos de la dislexia (véase Ramus, 2003 y Serniclaes, 2011 respectivamente). En tercer lugar, las tareas de identificación y discriminación permiten testar una hipótesis que se reivindica como específica de la dislexia: la hipótesis del modo de percepción alofónico (Serniclaes et al., 2004. Para una revisión de las principales medidas relativas a la percepción categorial, incluida la medida de la alofonía, véase Serniclaes (2006). El potencial de estas pruebas para poner de manifiesto diferencias sutiles incluso entre adultos normolectores queda ejemplificado en dos publicaciones recientes procedentes de nuestro laboratorio (López-Zamora, Luque, Álvarez y Cobos, en prensa; Luque, Luque y López-Zamora, 2011). La única hipótesis que quedaría fuera del rango de estas pruebas sería la reciente propuesta de Goswami (2003).

En resumen, desarrollar pruebas de identificación, discriminación y secuencia, y producir valores normativos o referenciales parece sólidamente justificado, tanto desde el punto de vista teórico, como por la amplitud de medidas que pueden generar. Además, parece que existe acuerdo en que si los déficit auditivo perceptivos aparecen, actúan como un factor agravante (Ramus, 2003). Por tanto, si estos déficit fueran o no específicos de la dislexia, esto no haría que perdieran su interés como instrumento clínico capaz de identificar perfiles diferenciados. 


\section{Método}

\section{Participantes}

La muestra estuvo constituida por alumnos de $2^{\circ}$ y $4^{\circ}$ de Primaria. Los participantes procedían de cuatro centros públicos, situados en zonas rurales y urbanas de Málaga. Se aplicó el PROLEC a 164 sujetos, seleccionando una muestra de 63 participantes (35 niños; $M=109.48$ ), que fueron clasificados en tres grupos: (1) grupo experimental con dificultad lectora $(n$ $=21 ; M=116.24 ; D T=3.25)$, (2) grupo control edad $(n=21$; $M=118.76 ; D T=3.80)$, y (3) grupo control nivel lector $(n=$ 21; $\mathrm{M}=93.43 D T=2.94)$. El grupo experimental tenían una puntuación de percentil $\leq 5$ en el test PROLEC. Igualmente, se les pasó el test de inteligencia (K-BIT). Fueron excluidos de la muestra los niños con las siguientes características: repetidores, extranjeros, provenientes de otros centros escolares, sujetos con problemas asociados (auditivos, visuales, cognitivos, psíquicos).

\section{Materiales}

Se utilizaron los siguientes materiales: (1) Test estandarizado de habilidades lectoras (PROLEC, Cuestos, Rodríguez y Ruano, 1996); (2) Test de Inteligencia K-BIT; (3) Prueba de Conciencia Fonémica PCF (Jiménez, 1995), únicamente conocimiento intrasilábico y segmental; (4) Pruebas de procesamiento auditivo rápido.

A continuación se presenta una breve explicación de los tres tipos de tareas de percepción auditiva: identificación, discriminación y secuencia (juicio de orden temporal).

\section{Prueba de identificación de sílabas oclusivas: continuo} BA-DA

En la Figura 1 pueden observarse la estructura de una prueba de identificación. Los estímulos son 9 sílabas del continuo /ba-da/. Los estímulos se construyeron manipulando los formantes 2 y 3 que distinguen los contrastes relativos al punto de articulación. En cada ensayo se presenta un estímulo y se pide al sujeto que lo clasifique en uno categoría: BA vs. DA. Son un total de 54 ensayos, con 6 ensayos de cada estímulo.

Figura 1. Estructura gráfica de la prueba de identificación, desarrollada a partir del original de Reed (1989) que hemos implementado.

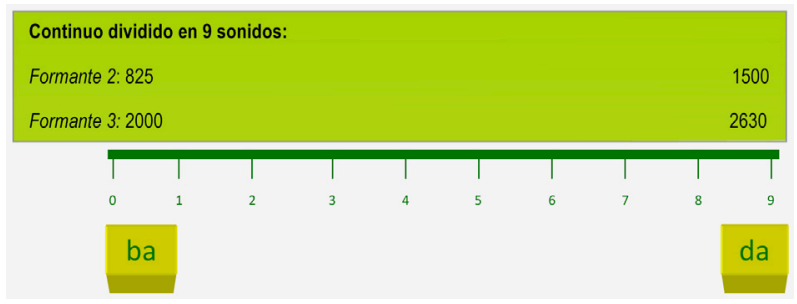

Prueba de discriminación de pares mínimos de sílabas oclusivas: continuo BA-DA

En la figura 2 puede observarse la estructura de una prueba de discriminación. Se pide al sujeto que interprete si las dos sílabas que se han presentado son iguales o diferentes. Todos los pares de sílabas se presentan con un intervalo entre estímulos (ISI) de 1 segundo. Cuando las sílabas son diferentes, la segunda sílaba está a dos pasos hacia la izquierda o derecha en el continuo /ba-da/. Hay un total de 90 ensayos, con 27 pares idénticos ( 3 veces cada par) y 63 pares diferentes (hay un total de 7 pares diferentes y se presentan 9 veces).

Figura 2. Estructura gráfica de la prueba de discriminación original de Reed (1989).

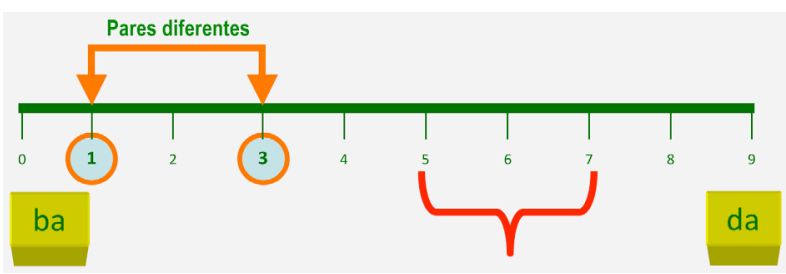

Pruebas de juicio de orden temporal de sílabas oclusivas

Estas pruebas se utilizan tanto con sílabas como con tonos, para evaluar la especificidad lingüística o no del déficit (véase Reed, 1989 o Manis y cols. 1997). Necesitan sólo de dos o tres sonidos diferentes (sílabas o tonos) que se presentan en sus cuatro combinaciones posibles (AA, AB, BA y BB). Se compone de una fase de habituación, una fase de entrenamiento en reconocer cada uno de los 2 sonidos, una fase de entrenamiento en orden temporal y una fase de test. En la fase de test, el ISI disminuye a medida que avanza la prueba $(400,300,150,50$, $10 \mathrm{~ms})$

Figura 3. Estructura gráfica de las pruebas de juicio de orden temporal originales de Reed (1989).

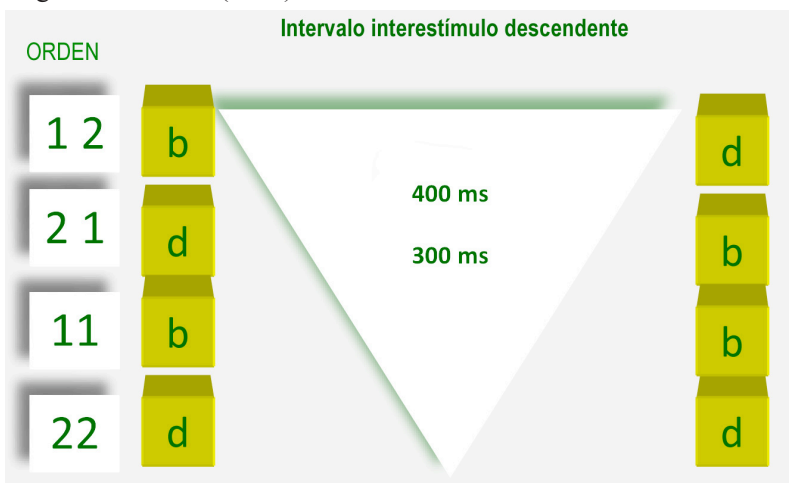

\section{Procedimiento}

Inicialmente se realizó una entrevista con los profesores y el Equipo de Orientación, con el fin de descartar de nuestra muestra a los sujetos con las siguientes características: repetidores, extranjeros, provenientes de otros centros escolares, sujetos con problemas asociados (auditivos, visuales, de aprendizaje, cognitivos, psíquicos. En segundo lugar, ser pasó la batería PROLEC a 164 alumnos. A los 63 seleccionados se les aplicó el K-BIT, con el fin de eliminar de la muestra a los sujetos con un déficit cognitivo. A continuación, 
fueron pasadas las pruebas de conciencia fonémica recogidas en Jiménez González (1995). Posteriormente, se aplicaron las pruebas experimentales de procesamiento auditivo rápido. Estas se dividían en tres tipos: las de identificación (I), discriminación (D) y secuencia (S), permutadas en sus tres posibles posiciones. Cada una de las pruebas estaban precedidas de ejemplos para asegurarnos que el sujeto había comprendido las instrucciones necesarias para la realización de la tarea.

\section{Resultados}

\section{Comparación de medias}

Se realizó un análisis de varianza de un factor sobre cada una de las pruebas de conciencia fonológica, mostrando todas las pruebas diferencias significativas. Los análisis posteriores evidenciaron que las discrepancias entre grupos siempre fueron entre el grupo experimental y los controles, no encontrándose diferencias significativas en ningún caso entre los grupos control. En la tabla 1 aparece un resumen de los resultados.

Tabla 1. Análisis de varianza de las pruebas de Conciencia Fonológica y diferencias entre grupos.

\begin{tabular}{lcccccc}
\hline Prueba & $\mathbf{F}(\mathbf{2}, \mathbf{6 0})$ & $\mathbf{p}$ & $\begin{array}{c}\text { Experimental } \\
\text { vs Control } \\
\text { Edad }\end{array}$ & $\begin{array}{c}\text { Experimental } \\
\text { vs Control Nivel } \\
\text { lector }\end{array}$ & $\begin{array}{c}\text { Control Edad } \\
\text { vs Control Nivel } \\
\text { lector }\end{array}$ \\
\hline CAHAP & 7.716 & .001 & .001 & .071 & .308 \\
CAHBP & 11.713 & .000 & .000 & .001 & .827 \\
CAHCP & 23.948 & .000 & .000 & .000 & .956 \\
CAHDP & 18.486 & .000 & .000 & .000 & .597 \\
CAISP & 14.194 & .000 & .000 & .000 & .979 \\
CAIAP & 16.462 & .000 & .000 & .000 & .970 \\
CAIGP & 28.522 & .000 & .000 & .000 & .951 \\
CAIOP & 26.555 & .000 & .000 & .000 & .801 \\
CAIVCP & 16.845 & .000 & .000 & .000 & .960 \\
CAIORP & 38.925 & .000 & .000 & .000 & .903 \\
CAIOMP & 38.925 & .000 & .000 & .000 & .901 \\
\hline
\end{tabular}

Las pruebas de procesamiento auditivo fueron sometidas, en primer lugar, a análisis de varianza, bien de un factor, bien de medidas repetidas con el objetivo de replicar los resultados obtenidos en otros laboratorios.

Las puntuaciones directas de la prueba de identificación fueron recopiladas de la siguiente forma. El programa registraba cada vez que el sujeto daba como respuesta DA con un punto (1) mientras la respuesta BA era codificada como cero (0). De esta forma las respuestas tomaron la forma de una curva sigmoidal, es decir, en el inicio, cuando la mayoría de las respuestas son $\mathrm{BA}$, la curva está cercana a cero, mientras que cuando la mayoría de las respuestas son DA, la curva está en su máximo nivel. Las puntuaciones directas se sometieron a un análisis de regresión a una función sigmoi- dal ofreciendo dos medidas independientes, límite categorial y pendiente. Para calcular los parámetros de límite categorial y pendiente de las curvas de identificación se construyó un programa en lenguaje Visual Basic. Se realizó un ANOVA por cada una de estas medidas, comparando los tres grupos que no resultó en ningún caso significativa. En la figura 4 se puede observar la curva de un sujeto tomado del grupo control edad.

Figura 4. Curva normalizada de un sujeto control edad. La identificación muestra hasta el estímulo cuatro una categorización BA. A partir del estímulo 6 el sujeto se estabiliza en la respuesta DA. La pendiente de identificación es muy acusada y el límite categorial se sitúa alrededor del valor 5, sesgado a la derecha, replicando los resultados de Reed (1989).

\section{Grupo control igualados en edad}

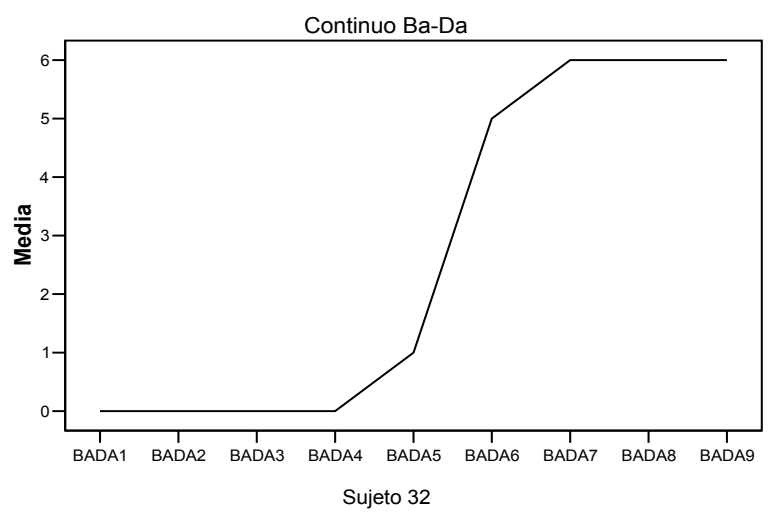

Con la prueba de discriminación se llevó a cabo un análisis factorial de medidas repetidas, siendo la variable intersujetos los tres grupos y la variable intrasujeto los siete pares de discriminación con sílabas diferentes. Los resultados fueron los siguientes: el factor pares resultó significativo $(F(6,55)$ $=40.037 ; p \leq .0001)$, pero no el factor grupo. La interacción pares $\mathrm{x}$ grupos resultó significativa $(F(12,112)=1.836, \mathrm{p} \leq$ .043). Aparecieron diferencias significativas entre grupos en los pares extremos. Los pares 1, 2 y 7 alcanzaron la significación $(F(2,60)=3.485$ у $p=.039 ; F(2,60)=3.365$ у $p$ $=.043 ; F(2,60)=3.313$ у $p=.045$, respectivamente). Estas diferencias se concretaron en los pares extremos, 1 y 7 , entre el grupo experimental y el grupo control nivel lector ( $p$ $=.041 \mathrm{y} .048$ respectivamente); entre el grupo experimental y el control edad en el par $2(p=.044)$. No se encontró ninguna diferencia entre los grupos control. Como puede observarse en la figura 7, las diferencias significativas se concentran en los extremos, es decir, hay diferencias de discriminación a favor del grupo experimental en pares que no marcan límites fonéticos. El gráfico de interacción muestra como la percepción categorial alcanza curvas menos pronunciadas en su parte central en el grupo experimental. Asimismo, representa las diferencias significativas encontradas en los pares extremos 1,2 y 7 . 
Figura 7. Representación de las curvas de discriminación de los pares diferentes en los tres grupos experimentales. Puede observarse junto a la curva menos pronunciada la mayor discriminación en los extremos característica de los disléxicos.

Graficas de Discriminación

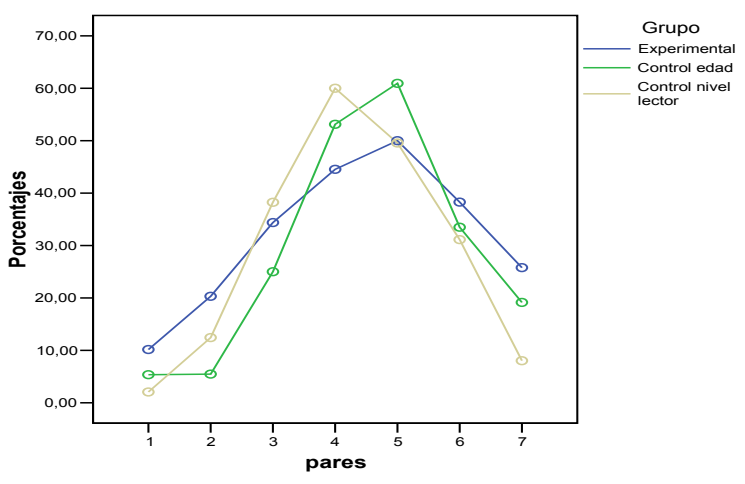

Se realizó un ANOVA sobre dos medidas de la prueba de secuencia, aciertos en los pares diferentes y aciertos en los pares iguales por grupos. Ambos análisis arrojaron resultados significativos, en los pares diferentes con una $F(2,60)=6.223$ y $p$ $=.004 \mathrm{y}$ en los pares iguales $F(2,60)=5.451 \mathrm{y} p=0.008$. No hubo diferencias significativas según el factor ISI. Las pruebas post hoc de Scheffé para los pares diferentes mostraron diferencias significativas entre el grupo experimental y los dos grupos controles (control edad, $p=.006$, y control nivel lector, $p=.046$ ). No hubo diferencias significativas entre los grupos controles. Del mismo modo para los pares iguales los resultados fueron los mismos, hubo diferencias significativas entre el grupo experimental y el grupo control edad $(p=.016)$ y entre el grupo experimental y el grupo control igualado en nivel lector $(p=.035)$, no habiendo diferencias entre los grupos controles.

\section{Criterio de desviación}

En segundo lugar se siguió el procedimiento de análisis de perfiles desviados común a estas investigaciones, siguiendo exactamente las especificaciones de Ramus y cols. (2003). Según Ramus un procedimiento común en este tipo de investigación es establecer un criterio de desviación basándose en la media y desviación típica. El proceso primero calcula la media y desviación típica de cada grupo control y elimina a aquellos sujetos de los grupos controles que exceden 1.65 de desviación sobre la media. Una vez eliminados estos falsos positivos se recalcularon las puntuaciones típicas de las puntuaciones para toda la muestra, lo que permitió distinguir aquellos sujetos del grupo experimental que estaban fuera del criterio. Según Ramus este proceso produce aproximadamente un falso positivo por cada medida. En nuestro caso tuvimos 5 casos en el grupo control edad y tres en el grupo control nivel lector. Retiramos de la muestra los cinco sujetos del grupo control edad, más cinco del grupo control nivel lector, añadiendo dos que estaban por encima de 1.5 desviaciones típicas, un criterio utilizado en otros estudios. Esto igualó los grupos controles en 16 sujetos.
El grupo experimental fue también recortado siguiendo el criterio de quitar aquellos sujetos con una puntuación más alta en el PROLEC, es decir, los que mostraban un retraso lector sensiblemente menor.

Posteriormente se procedió al análisis según el criterio de desviación. En la tabla 2 se reflejan en negrita las puntuaciones que superan 1.65 desviaciones típicas, mientras se representan en gris las que sólo superan 1.5 desviaciones. Para la prueba de identificación se consideraron los parámetros de límite categorial y pendiente. Las figuras 5 y 6 muestran ejemplos de puntuaciones fuera de criterio, bien debido a su límite categorial bien a su pendiente. En el caso de la prueba de discriminación, tomamos los extremos del continuo como medida, cuatro pares en total, de acuerdo con los resultados significativos obtenidos en la interacción pares por grupos. En la prueba de secuencia o juicio de orden temporal se establecieron como medidas los pares iguales por un lado y los diferentes por otro. El ISI no fue considerado dado el resultado no significativo del análisis de varianza.

Tabla 2. Aplicación del criterio de desviación a las medidas derivadas de las pruebas de identificación, discriminación y secuencia. Relación de estas medidas con la conciencia fonológica.

\begin{tabular}{|c|c|c|c|c|c|c|c|c|c|}
\hline \multirow{2}{*}{$\begin{array}{c}\mathrm{N}^{\mathbf{o}} \\
\text { sujeto }\end{array}$} & \multicolumn{2}{|c|}{ Identificación } & \multicolumn{4}{|c|}{ Discriminación } & \multicolumn{2}{|c|}{ Secuencia } & \multirow{2}{*}{$\begin{array}{c}\begin{array}{c}\text { Puntuación } \\
\text { en CF }\end{array} \\
X=78.80\end{array}$} \\
\hline & Límite & pendiente & BD02 & BD13 & BD57 & BD68 & Diferentes & Iguales & \\
\hline 13 & & & 2.66 & & & & -1.97 & & 59.50 \\
\hline 3 & & & & & & & & & 63.25 \\
\hline 7 & 2.34 & & & & & & -2.65 & -3.61 & 65.75 \\
\hline 14 & & -1.84 & 1.57 & 2.93 & 1.56 & 1.96 & & & 67.00 \\
\hline 4 & & & & & & 2.52 & -5.14 & -4.17 & 68.25 \\
\hline 10 & & & & & & & & & 68.75 \\
\hline 15 & 1.56 & -2.07 & 2.66 & 3.66 & & 1.96 & & & 75.75 \\
\hline 2 & & & & & & & & & 80.00 \\
\hline 11 & & & & & & & & & 80.75 \\
\hline 8 & & & & & & & & & 85.75 \\
\hline 5 & 3.56 & -1.50 & & & 1.55 & 2.52 & & -3.05 & 88.25 \\
\hline 12 & & & & & & & & & 88.75 \\
\hline 9 & & & & & 2.05 & & & & 90.00 \\
\hline 16 & & & & & & & & & 90.00 \\
\hline 1 & & & & & & & & & 91.25 \\
\hline 6 & -1.77 & & & 2.19 & & & & & 91.25 \\
\hline
\end{tabular}

Figura 5. La grafica muestra un sujeto experimental que presenta un límite categorial desviado.

Grupo con dificultades lectoras

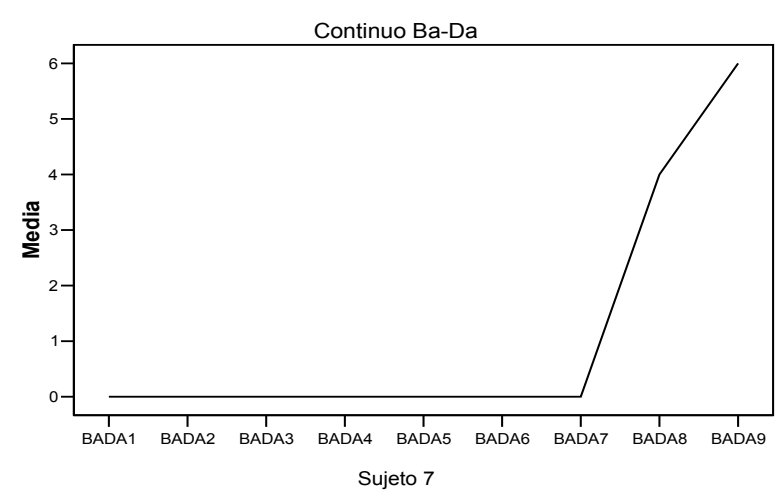


Figura 6. La grafica muestra la falta de pendiente del el sujeto 15, perteneciente al grupo experimental.

Pruebas de identificación

\section{Grupo con dificultades lectoras}

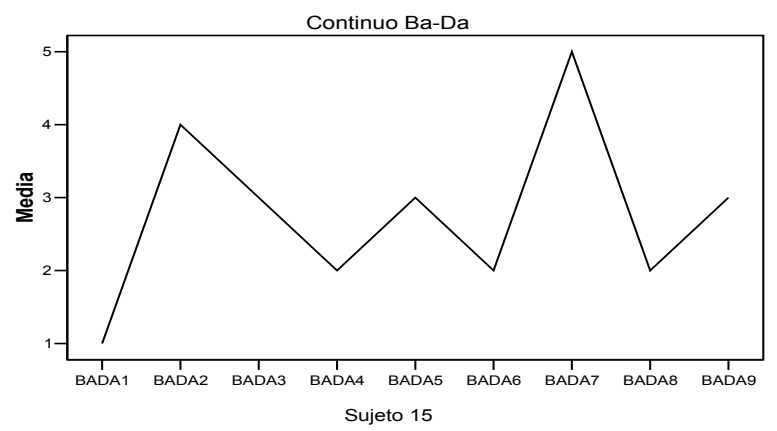

Aplicando el criterio de que un sujeto debe ser identificado al menos por dos pruebas, de un total de 16 sujetos fueron identificados hasta 7 , es decir, el $44 \%$ por ciento de la muestra. Se alcanza el $50 \%$ si consideramos al sujeto 9 , que sólo fue marcado por una medida.

Para estudiar la relación entre la conciencia fonológica y las pruebas de procesamiento auditivo se instrumentó un procedimiento inspirado en Manis et al. (1997). Se confeccionó una medida global de conciencia fonológica, tomando únicamente a los participantes del grupo experimental. La medida se confeccionó a partir de cuatro pruebas, dado que sólo éstas presentaban una desviación típica suficiente para marcar diferencias, superior a dos. Con estas cuatro pruebas confeccionamos una medida global de conciencia fonológica (CAHAP, CAIVCP, CAIORP, CAIOMP). Dado el número escaso de sujetos para otros análisis, nos limitamos a señalar que existe una mayor proporción de sujetos con desviación en las pruebas de procesamiento auditivo situados por debajo de la puntuación media en conciencia fonológica.

\section{Discusión}

Tal y como mencionábamos en la introducción la replicación de los resultados obtenidos en otros laboratorios era una condición necesaria para considerar el uso de las medidas de procesamiento auditivo rápido y percepción categorial como elemento con valor diagnóstico. Los diferentes análisis de varianza han producido, en general, resultados similares a los de la bibliografía previa.

El análisis de varianza de los parámetros asociados a la tarea de identificación, pendiente y límite categorial, no resultaron significativos. En general, estos resultados permiten observar la debilidad de la percepción categorial en sujetos disléxicos. La pendiente muestra la agudeza del sistema de categorización mientras que el desplazamiento del límite categorial nos muestra la organización del espacio de representación (proporcionalidad). Tampoco se alcanza la signifi- cación en el estudio de Reed (1989) respecto a la medida de la pendiente (del límite categorial no se proporciona información). Sí aparecen perfiles interesantes en la medida de desviación que comentaremos más adelante. Por otra parte, la identificación exige la categorización explícita y consciente del estímulo, mientras que la prueba de discriminación sólo demanda distinguir entre pares de sonidos iguales versus diferentes. De hecho, propiamente, la medida de la percepción categorial (Liberman, Harris, Hoffman y Griffith, 1957) implica comprobar el ajuste entre la función de discriminación que puede predecirse a partir de las puntuaciones de identificación frente a las puntuaciones reales observadas mediante la prueba de discriminación. Normalmente el desajuste consiste en que las puntuaciones predichas están por encima de la discriminación real observada. Por esta razón, la prueba de discriminación suele presentar mayor sensibilidad a las diferencias. Así, ha sido en nuestro caso, donde la prueba de discriminación ha arrojado una interacción entre pares y grupos basada en la falta de estabilidad de la respuesta en los pares extremos del grupo de disléxicos (ver figura 7). Este resultado, junto con una curva de discriminación más achatada son los resultados característicos de los grupos disléxicos (Serniclaes, 2011).

Por su parte las pruebas de secuencia o juicio de orden temporal poseen otra naturaleza. Aunque fueron propuestas por Tallal (1980) en el contexto de la hipótesis sobre el procesamiento auditivo rápido, su naturaleza exacta ha sido objeto de una fuerte polémica. La hipótesis de Tallal posee dos versiones que bien podrían ser muy diferentes. Por un lado, se afirma que la dificultad de los disléxicos podría originarse en su limitada sensibilidad para percibir los sutiles cambios que se producen en, por ejemplo, los formantes dos y tres de los contrastes de punto de articulación. Se ha argumentado que la percepción de la ratio, es decir, la percepción de las rápidas transiciones en estos formantes (cambios de frecuencia), dentro de los primeros 35 milisegundos, estaría alterada, de forma que el disléxico tendría dificultades para distinguir los sonidos a lo largo de este continuo (p.e.: /b/-/d/-/g/). Sin embargo, las pruebas de secuencia parecen medir un fenómeno diferente, relacionado con la ratio de percepción, es decir, el tiempo necesario que debe separar una secuencia de estímulos para que estos sean adecuadamente percibidos. En este sentido, nuestros resultados han sido poco favorables a esta segunda posibilidad, ya que no ha aparecido ni efecto principal del factor ISI, ni ninguna otra interacción con este factor. Más aún, el hecho de que hayan aparecido sendas interacciones entre los factores "secuencias iguales" y "secuencias diferentes", y el factor grupo, lleva a interpretar los resultados como problemas de identificación de los sonidos más que como dificultades con su secuencia. Esta interpretación de las pruebas de secuencia y su debate correspondiente no es tampoco una novedad (Mody, Studert-Kennedy y Brady, 1997). Sin embargo, estos resultados no replican los presentados por Reed (1989). 
En cualquier caso, el hecho de que las diferencias perceptivas sean sutiles y difíciles de detectar no hace que pierdan ni su interés teórico ni tampoco su valor diagnóstico. Tal y como argumentaban Galaburda, LoTurco, Ramus, Fitch, y Rosen (2006), en la mayoría de los desórdenes del desarrollo existen constelaciones de síntomas que cambian con la maduración, otros permanecen inmutables, algunos mejoran y otros empeoran. Esta complejidad hace imposible detectar e identificar ciertas asociaciones de síntomas, incluso relaciones causales, pasadas las primeras etapas del desarrollo. Según Goswami (2003), del hecho de que los déficits de bajo nivel no puedan ser detectados con facilidad en adultos que han recibido tratamiento, no debe concluirse que estos déficits auditivos no tengan una influencia critica en el desarrollo fonológico. En otras palabras, es una conjetura razonable mantener que existe tal relación. Que ese déficit sea aún detectable todavía a los 9 años podría ser significativo en el perfil diagnóstico de ese individuo.

Esta última idea resume el objetivo principal de nuestro estudio: desarrollar estas pruebas para testarlas en el contexto real de un proceso de evaluación de niños con dificultades de lectoescritura. Por tanto, de forma ecléctica, sin discutir la especificidad o no de estas medidas, puede afirmarse que los resultados son muy satisfactorios, en cuanto a las medidas del criterio de desviación, la principal herramienta clínica presente en este trabajo.

Primero, tal y como puede observarse en la tabla 2, el número de sujetos que poseen alguna medida fuera de criterio es del $50 \%$, del $44 \%$ cuando se exige que sean al menos dos las puntuaciones fuera de criterio. Hay además que matizar algunos aspectos. En primer lugar, algunos estudios que han alcanzado este techo de desviaciones, utilizaban más pruebas, medidas y además se han realizado con adultos (Ramus et al, 2003). Luego el nivel de identificación ha sido muy alto. En segundo lugar, estas identificaciones están presentadas en la tabla 2 con dos criterios, 1.65 y 1.5 desviaciones típicas, ambos criterios exigentes. En el contexto de evaluaciones individualizadas, el clínico podría utilizar, según criterio experto, otros valores menos exigentes; dependiendo del cuadro de síntomas o historial ante el que se encuentre. Al margen de la estadística inferencial (basada en poblaciones), las gráficas 5 y 6 muestran que algunos perfiles individuales son lo suficientemente desviados como para tomar en consideración estas variables.

Hay que admitir, sin embargo, que ninguna prueba auditiva parece mostrar un perfil destacado. Tampoco era el objetivo de estudio resolver o aportar algo significativo al que se ha mostrado como un problema altamente complejo: la naturaleza exacta, específica, del déficit perceptivo. En cualquier caso, nuestros resultados sí dejan de nuevo constancia de la relación entre conciencia fonológica y aprendizaje de la lectura: las dificultades en la identificación y manipulación consciente de los fonemas es el mejor predictor de las dificultades de aprendizaje de la lectura.
Muchos autores han estudiado la relación entre la percepción de estos fonemas y su manipulación consciente. Manis et al (1997), revisaron la bibliografía que estudiaba esta relación, y ellos mismo encontraron que existe una mayor proporción de sujetos identificados entre aquellos que poseen peor conciencia fonológica. Sin defender el valor causal de estas asociaciones, creemos de interés clínico que las dificultades de percepción del habla aparezcan proporcionalmente más representadas en el contexto de baja conciencia fonológica, aunque no en exclusiva, también en nuestros resultados. Por otra parte, las pruebas de procesamiento auditivo rápido fueron las tareas sobre las que se sustentó la primera hipótesis de déficit fonológico que relacionó la dislexia con los trastornos específicos del lenguaje (TEL; Tallal, 1980). Ha recibido una reciente revisión por parte de su autora (Tallal, 2003, 2004) y son objeto de un fructífero debate, plagado de alternativas, probablemente complementarias, que pueden revisarse en un reciente número especial de una destacada revista (Messaoud-Galusi y Marshall, 2010).

Con todas las limitaciones y dificultades comentadas, parece posible proponer que estos resultados son un fundamento más para defender un procedimiento que instrumente estas pruebas como parte del proceso diagnóstico de la dislexia y otros síntomas o trastornos comórbidos. Desde luego, las pruebas de procesamiento auditivo y percepción categorial no poseen por si solas valor diagnóstico. Sin embargo, sí pueden aportar información significativa en el contexto de una evaluación que vaya más allá de la simple medición de las habilidades de lectura y escritura. Una evaluación que incluya pruebas críticas de conciencia fonológica y otras pruebas derivadas de la hipótesis fonológica e hipótesis relacionadas, tal y como defienden Carrillo y Alegría (2009). En el contexto de un diagnóstico de este tipo, las pruebas de procesamiento auditivo rápido y/o percepción categorial pueden contribuir a contrastar la desproporción que presentan los disléxicos entre sus habilidades cognitivas generales y las relativas a la fonología del lenguaje hablado y escrito.

\section{Referencias}

1. Boets, B., Wouters, J., van Wieringen, A. y Ghesquière, P. (2007). Auditory processing, speech perception and phonological ability in pre-school children at high-risk for dyslexia: A longitudinal study of the auditory temporal processing theory. Neuropsychologia, 45, 1608-1620. http://dx.doi. org/10.1016/j.neuropsychologia.2007.01.009

2. Bowers, P. G. y Swanson, L. B. (1991). Naming speed deficits in reading disability: Multiple measures of a singular process. Journal of Experimental Child Psychology, 51, 195-219. http://dx.doi.org/10.1016/0022-0965(91)90032-N

3. Bradley, L. y Bryant, P. (1978). Difficulties in auditory organization as a possible cause of reading backwardness. Nature, 271, 746-747. http://dx.doi.org/10.1038/271746a0

4. Brandt, J. y Rosen, J. J. (1980). Auditory phonemic perception in dyslexia: Categorical identification and discrimina- 
tion of stop consonants. Brain and Language, 9, 324-337. http://dx.doi.org/10.1016/0093-934X(80)90152-2

5. Bruck, M. (1992). Persistence of dyslexics' phonological awareness deficits. Developmental Psychology, 28, 874886. http://dx.doi.org/10.1037/0012-1649.28.5.874

6. Byrne, B. y Shea, P. (1979). Semantic and phonetic memory codes in beginning readers. Memory and Cognition, 7, 333-338. http://dx.doi.org/10.3758/BF03196936

7. Carrillo, M.S. y Alegría, J. (2009). Exploración de habilidades fonológicas en escolares disléxicos: teoría y práctica. Revista de Logopedia, Foniatría y Audiología, 29, 105-120.

8. Carrillo, M. S., Alegría, J., Miranda, P. y Sánchez, N. (2011). Evaluación de la dislexia en la escuela primaria: Prevalencia en español. Escritos de Psicología,4. DOI: 10.5231/psy.writ.2011.1407

9. Cuetos, F., Rodríguez, B. y Ruano E. (2000). Evaluación de los procesos lectores. PROLEC. Madrid: TEA.

10. Denckla, M. B. y Rudel, R. G. (1976). Rapid automatized naming (R.A.N.): Dyslexia differentiated from other learning disabilities. Neuropsychologia, 14, 471-479. http://dx.doi.org/10.1016/0028-3932(76)90075-0

11. Deno, S. L. (1987). Special focus: Curriculum-based measurement. Teaching Exceptional Children, 20, 41.

12. Fletcher, J., Shaywitz, S., Shankweiler, D., Katz, L., Liberman, I., Stuebing, K. K., Francis, D.J., Fowler, A.E. y Shaywitz, B.A. (1994). Cognitive profiles of reading disability: comparisons of discrepancy and low achievement definitions. Journal of Educational Psychology, 86, 6-23. http://dx.doi.org/10.1037/0022-0663.86.1.6

13. Fuchs, D. y Fuchs, L. S. (2006). Introduction to response to intervention: What, why, and how valid is it? Reading Research Quaterly, 41, 93-99. http://dx.doi.org/10.1598/ RRQ.41.1.4

14. Galaburda, A. M., LoTurco, J., Ramus, F., Fitch, R. H. y Rosen, G. D. (2006). From genes to behavior in developmental dyslexia. Nature Neuroscience, 9, 1213-1217. http://dx.doi.org/10.1038/nn1772

15. Goswami, U. (2003). Why theories about developmental dyslexia require developmental designs. TRENDS in Cognitive Sciences, 7, 534- 540. http://dx.doi.org/10.1016/j. tics.2003.10.003

16. Jiménez, J. E. (2010). Response to intervention (RtI) Model: A promising alternative for identifying students with learning disabilities? Psicothema, 22, 932-934.

17. Jiménez, J. E., Baker, D. L., Rodríguez, C., Crespo, P., Artiles, C. y Alfonso, M. (2011). Evaluación del progreso de aprendizaje en lectura dentro de un Modelo de Respuesta a la Intervención (RtI) en la Comunidad Autónoma de Canarias. Escritos de Psicología, 4. DOI: 10.5231/psy. writ.2011.1207

18. Jiménez González, J. E (1995). Conciencia fonológica y aprendizaje de la lectura: Teoría, evaluación, e intervención. Madrid: Síntesis.
19. Kaufman, A. S. y Kaufman, N. L. (1997). K-BIT: Test breve de inteligencia de Kaufman. Madrid: TEA.

20. Kuhl, P. (2004). Early Language acquisition: cracking the speech code. Nature Reviews, Neuroscience, 5, 831-843. http://dx.doi.org/10.1038/nrn1533

21. Liberman, A. M., Harris, K. S. Hoffman, H. S. y Griffith, B. C. (1957). The discrimination of speech sounds within and across phoneme boundaries. Journal of Experimental Psychology, 54, 358-368. http://dx.doi.org/10.1037/h0044 $\underline{417}$

22. López-Zamora, M., Luque, J.L., Álvarez, C. y Cobos, P. L. (en prensa). Individual differences in categorical perception are related to sub-lexical/phonological processing in reading. Scientific Studies of Reading.

23. Luque, D., Luque, J. L. y López-Zamora, M. (2011). Individual Differences in pseudohomophony effect are relates to auditory categorical perception skills. Learning and Individual Differences, 21, 210-214. http://dx.doi. org/10.1016/j.lindif.2011.01.002

24. Luque, J. L., López-Zamora, M. y Álvarez (2011). Beyond decoding deficit: Inhibitory effect of positional syllable frequency in dyslexic spanish children. Eighteenth Annual Conference of the Society for the Scientific Study of Reading, Florida (EE.UU), 2011.

25. Luque, J.L., Serniclaes, W., López-Zamora, M., Bordoy, S., Giménez, A.,Rosales, V. y Varona, S. (2011). Exploring Categorical Perception Deficit in Spanish Dyslexics Children. 10th International Symposium of Psycholinguistics. Donostia, Spain.

26. Lyon, G., Shaywitz, S. y Shaywitz, B. (2003). A definition of dyslexia. Annals of Dyslexia, 53, 1-14. http:/dx.doi. org/10.1007/s11881-003-0001-9

27. Lyytinen, H.,Ahonen, T., Eklund, K., Guttorm, T., Kulju, P., Laakso, M. L., Leiwo, M., Leppanen, P., Lyytinen, P. Poikkeus, A. M., Richardson, U, Torppa, M. y Viholainen, H. (2004). Early development of children at familial risk for dyslexia-follow-up from birth to school age. Dyslexia, 10, 146-178. http://dx.doi.org/10.1002/dys.274

28. Manis, F. R, Custodio, R y Szeszulski, P. A. (1993). Development of phonological and orthographic skill: A 2-year longitudinal study of dyslexic children. Journal of Experimental Child Psychology, 56, 64-86. http://dx.doi. org/10.1006/jecp.1993.1026

29. Manis, F. R., McBride-Chang, C., Seidenberg, M., Keating, P., Doi, L. M., Munson, B. y Petersen, A. (1997). Are speech perception deficits associated with developmental dyslexia? Journal of Experimental Child Psychology, 66, 211-235. http://dx.doi.org/10.1006/jecp.1997.2383

30. Marzola E. y Shepherd M. 2005. Assessment of reading difficulties. En J.R. Birsh (Ed.), Multisensory teaching of basic language skills (pp. 171-85). Baltimore, MD: Brookes.

31. McBride-Chang, C. (1995). Phonological processing, speech perception, and reading disability: An integrative re- 
view. Educational Psychologist, 30, 109-121. http://dx.doi. org/10.1207/s15326985ep3003_2

32. Messaoud-Galusi, S. y Marshall, C. R. (2010). Special issue. Exploring the overlap between dyslexia and SLI: The role of phonology. Scientific Studies of Reading, 14, 1-7. http://dx.doi.org/10.1080/10888430903242076

33. Mody,M., Studdert_Kennedy, M. y Brady, S. (1997). Speech perception deficits in poor readers: Auditory processing or phonological coding? Journal of Experimental Child Psychology, 64, 199-231. http://dx.doi.org/10.1006/ jecp.1996.2343

34. Molfese, D. L. (2000). Predicting dyslexia at 8 years of age using neonatal brain responses. Brain and Language, 72, 238-245. http://dx.doi.org/10.1006/brln.2000.2287

35. Morris, R., Stuebing, K., Fletcher, J., Shaywitz, S.,Lyon, G., Francis, D.J. y Shaywitz, B.A. (1998). Subtypes of reading disability: coherent variability around a phonological core. Journal of Educational Psychology, 90, 347-73. http://dx.doi.org/10.1037/0022-0663.90.3.347

36. Pennington, B. F. (2006). From single to multiple deficit models of developmental disorders. Cognition, 101, 385413. http://dx.doi.org/10.1016/j.cognition.2006.04.008

37. Pernet, C., Dufor, O. y Démonet, J.F. (2011). Re-Defining Dyslexia: Accounting for Variabilit. Escritos de Psicología, 4. DOI: 10.5231/psy.writ.2011.2407

38. Ramus, F. (2003). Developmental dyslexia: specific phonological deficit or general sensorimotor dysfunction? Current Opinion in Neurobiology, 13, 1-7. http://dx.doi. org/10.1016/S0959-4388(03)00035-7

39. Ramus, F., Rosen, S., Dakin, S. C., Day, B.L., Castellote, J.M.,White, S. y Frith, U. (2003). Theories of developmental dyslexia: insights from a multiple case study of dyslexic adults. Brain, 126, 841-865. http://dx.doi.org/10.1093/ brain/awg076

40. Reed, M. A. (1989). Speech Perception and the Discrimination of Brief Auditory Cues in Reading Disabled Children. Journal of Experimental Child Psychology, 48, 270-292. http://dx.doi.org/10.1016/0022-0965(89)90006-4

41. Richardson, U., Leppänen, P. H. T., Leiwo, M. y Lyytinen, H. (2003). Speech perception of infants with high familial risk for dyslexia differ at the age of 6 months. Developmental Neuropsychology, 23, 385-397. http://dx.doi. org/10.1207/S15326942DN2303 5

42. Scarborough, H. S. (1990). Very early language deficits in dyslexic children. Child Development, 61, 1728-1743. http://dx.doi.org/10.2307/1130834

43. Serniclaes, W. (2006).Allophonic perception in developmental dyslexia. Origin, reliability and implications of the categorical perception deficit. Written Language \& Literacy, 9, 135-152. http://dx.doi.org/10.1075/w11.9.1.09ser

44. Serniclaes, W. (2011). Allophonic Perception in Dyslexia: An overview. Escritos de Psicología, 4. DOI: 10.5231/psy. writ.2011.2607
45. Shankweiler, D., Liberman, I. Y., Mark, L. S., Fowler, C. A., y Fischer, F. W. (1979). The speech code and learning to read. Journal of Experimental Psychology: Human Learning and Memory, 5, 531-545. http://dx.doi. org/10.1037/0278-7393.5.6.531

46. Shaywitz S, Fletcher J, Holahan J, Shneider A, Marchione K, et al. (1999). Persistence of dyslexia: The connecticut longitudinal study at adolescence. Pediatrics, 104, 135159. http://dx.doi.org/10.1542/peds.104.6.1351

47. Shaywitz, S. (2003). Overcoming dyslexia: A new and Complete Science-Based program for reading problems at any level. New York: Knopf.

48. Shaywitz, S., Morris, R., \& Shaywitz, B. (2008). The education of dyslexic children from childhood to young adulthood. Annual Review of Psychology, 59, 451-475. http:// dx.doi.org/10.1146/annurev.psych.59.103006.093633

49. Snowling, M.J. (2000). Dyslexia. Oxford: Blackwell.

50. Sprenger-Charolles, L. (2011). Dyslexia subtypes in languages differing in orthographic transparency: English, French and Spanish. Escritos de Psicología, 4. DOI: 10.5231/psy.writ.2011.1707

51. Sprenger-Charolles, L., Colé, P., Béchennec, D.y KipfferPiquard, A. (2005). French normative data on reading and related skills from EVALEC, a new computerized battery of tests (end Grade 1, Grade 2, Grade 3, and Grade 4). Revue Européenne de Psychologie Appliqué, 55, 157-186.

52. Stanovich, K. (1988). Explaining the differences between the dyslexic and the garden-variety poor reader: the phonological core variable difference model. Journal of Learning Disabilities, 21, 590-604. http://dx.doi. org/10.1177/002221948802101003

53. Stanovich, K. y Siegel, L. (1994). Phenotypic performance profile of children with reading disabilities: a regressionbased test of the phonological-core variable-difference model. Journal of Educational Psychology, 86, 24-53. http://dx.doi.org/10.1037/0022-0663.86.1.24

54. Tallal, P. (1980). Auditory temporal perception, phonics, and reading disabilities in children. Brain Language, 9, 182-198. $\quad$ http://dx.doi.org/10.1016/0093-934X(80)901 39-X

55. Tallal, P. (2003). Language learning disabilities: Integrating research approaches. Current Directions in Psychological Science, 12, 206-211. http://dx.doi.org/10.1046/j.09637214.2003.01263.x

56. Tallal, P. (2004). Improving language and literacy is a matter of time. Nature Reviews Neuroscience 5, 721-728. http://dx.doi.org/10.1038/nrn1499

57. Van Leeuwen, T., Been, P., Van Herten, M., Zwarts, F., Maassen, B. y Van der Leij, A. (2007). Cortical categorization failure in 2-month-old infants at risk for dyslexia. Neuroreport, 18, 857-861. http://dx.doi.org/10.1097/ WNR.0b013e3280c1e2bf

58. Wagner, R. K. y Torgesen, J. K. (1987). The nature of pho- 
nological processing and its causal role in the acquisition of reading skills. Psychological Bulletin, 101, 192-212. http:// dx.doi.org/10.1037/0033-2909.101.2.192

59. Werker, J. F. y Tees, R. C. (1987). Speech perception in severely disabled and average reading children. Canadian Journal of Psychology, 41, 48-61. http://dx.doi. org $/ 10.1037 / \mathrm{h} 0084150$

60. Wolf, M. (1986). Rapid alternating stimulus naming in the developmental dyslexias. Brain and Language, 27, 360379. http://dx.doi.org/10.1016/0093-934X(86)90025-8
61. Ziegler, J. C. y Goswami, U. (2005). Reading acquisition, developmental dyslexia, and skilled reading across languages: a psycholinguistic grain size theory. Psychological Bulletin, 131, 3-29. http://dx.doi.org/10.1037/00332909.131.1.3

Fecha de recepción: 14 de marzo de 2011

Fecha de recepción de la versión modificada: 5 de abril de 2011

Fecha de aceptación: 29 de abril de 2011 